\title{
Burst abdomen in pregnancy: A proposed management algorithm
}

\author{
Amalachukwu M. Okpala ${ }^{1}$, Samuel A. Debrah ${ }^{2}$ and Mohammed Mouhajer ${ }^{3}$ \\ Ghana Med J 2016; 50(2): 115-118 DOI: http://dx.doi.org/10.4314/gmj.v50i2.10 \\ ${ }^{1}$ Department of Plastic Surgery, Leeds General Infirmary, Leeds, United Kingdom ${ }^{2}$ Department of Surgery, \\ School of Medical Sciences, University of Cape Coast, Cape Coast, Ghana ${ }^{3}$ Department of Obstetrics and \\ Gynaecology, Korle Bu Teaching Hospital, Accra, Ghana
}

Corresponding author: Amalachukwu M. Okpala

E-mail:drmaluski@yahoo.com

Conflict of interest: None declared

\section{SUMMARY}

Management of the burst abdomen is complex due to the co-morbidities associated with it. When coupled with intraabdominal sepsis and pregnancy, it becomes even more difficult due to the ethical issues that have to be considered when managing both mother and child. Due to the paucity of literature on this subject, a management algorithm has been proposed which aims at tackling this delicate issue. However, the major consideration in the management of these cases is that decisions are to be made based on optimization of the condition of the mother.

Keywords: Burst abdomen, pregnancy, management, algorithm, intra-abdominal sepsis

\section{INTRODUCTION}

Acute abdomen in pregnancy, and thus management of its complications, remains one of the most challenging diagnostic and therapeutic dilemmas of today despite advancements in medical technology. ${ }^{1}$ Wound dehiscence following intra - abdominal sepsis in pregnancy is rare, and the management of such a complex case is a conundrum to both the surgeon and the obstetrician. Whether to close or not to close the abdomen, or when and how to deliver the fetus depends on both maternal and fetal factors which must be considered to have the most optimal outcome.

\section{Case report}

Eighteen-year-old Miss CN was referred from a hospital in the district to the Central Regional Hospital as a case of intestinal obstruction post laparotomy for typhoid perforation in pregnancy at 23 weeks gestation. Patient had undergone surgery 3 days prior to referral, and intra operative findings were faeculent peritoneal fluid, and a single large ileal perforation $15 \mathrm{~cm}$ from the ileo-caecal junction. A simple closure had been done. Three days post operatively, abdominal distension was noticed, associated with bilious vomiting, hence the referral from the district hospital.

On initial assessment at the emergency unit, a young, very ill looking female with marked pallor and a temperature of $38 .{ }^{\circ} \mathrm{C}$ was noted. She was tachypnoeic and tachycardic, with a blood pressure of $100 / 64 \mathrm{mmHg}$.
Her abdomen was distended, with pus oozing out from a sub-umbilical midline incision, which was held, together by non-absorbable sutures. Obstetric examination (abdomino-pelvic ultrasound) revealed a viable fetus at 23 weeks gestation.

Laboratory investigations showed a haemoglobin level of $6.6 \mathrm{~g} / \mathrm{dl}$ and a white cell count of $14.6 \times 10^{9} / \mathrm{L}$. A diagnosis of deep surgical site infection post laparotomy with severe anaemia was made. Patient was admitted and resuscitated with intravenous fluids, intravenous antibiotics and blood transfusions. A wound swab was taken for culture and sensitivity. Skin sutures were removed to facilitate drainage of pus, and patient was managed on a high protein/calorie enteral diet. On the tenth day of admission, the patient developed a burst abdomen. A Bogota bag was provided for closure of the abdomen due to the finding of a septic hostile abdomen in an anaemic malnourished patient.

The surgical wound granulated over rapidly, and the foetus was monitored regularly via abdominal ultrasound and daily foetal kick counts. Seven weeks after admission (gestational age 31 weeks) and after a course of steroids to promote foetal lung maturation, patient went into preterm labour and delivered a live male infant via spontaneous vaginal delivery. The neonate was taken to the neonatal intensive care unit for further monitoring. 


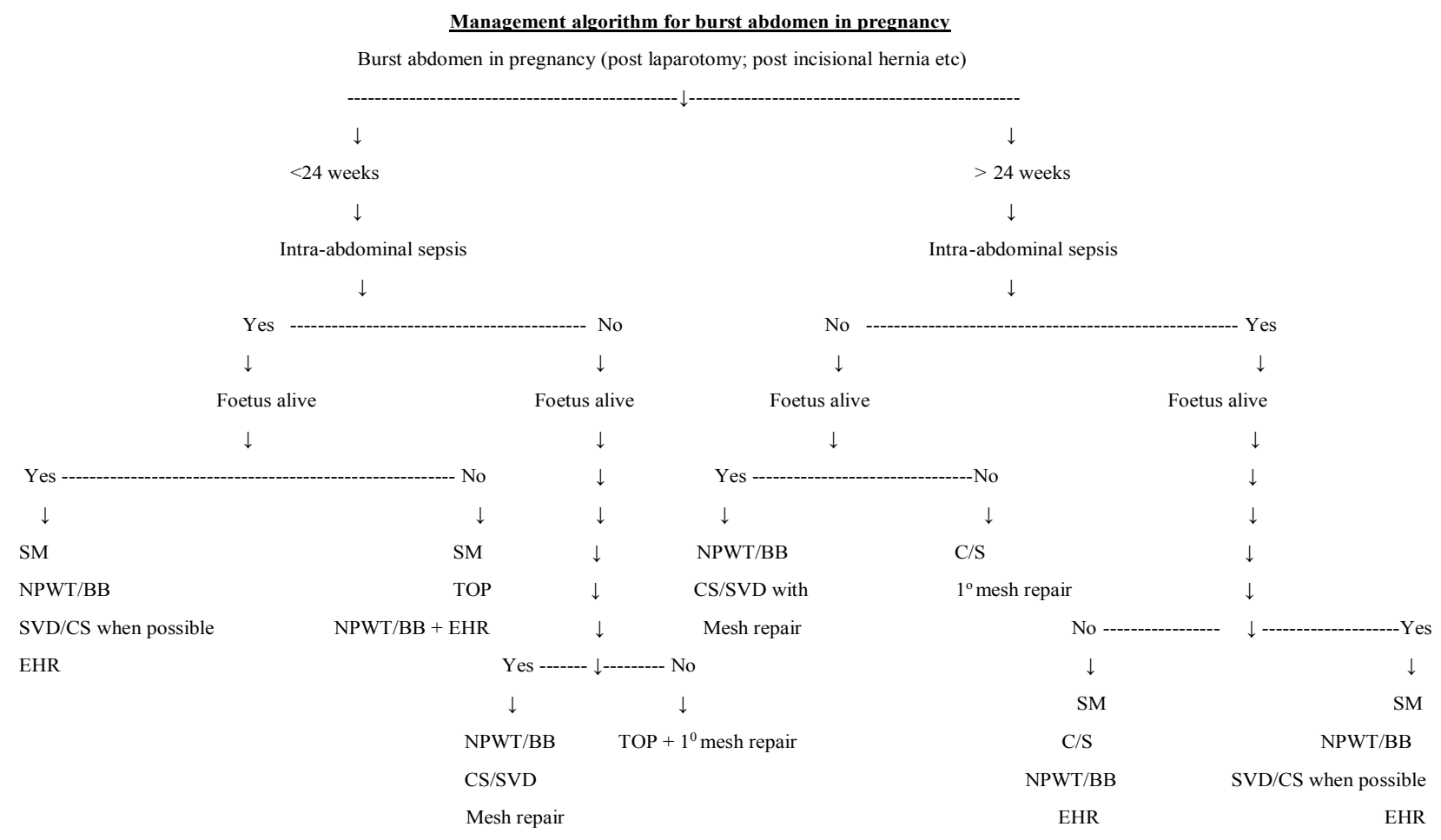

- $\quad$ SVD - Spontaneous Vaginal Delivery; NPWT - Negative Pressure Wound Therapy; CS - Caesarean Section; TOP - Termination of pregnancy

- SM -Surgical management involves laparotomy and peritoneal lavage if required; BB - Bogota bag;

- $\quad$ EHR -Elective hernia repair

Patient was discharged with a healthy neonate to be reviewed on an outpatient basis. The wound subsequently healed with secondary intention, with future consideration of incisional hernia repair.

\section{DISCUSSION}

Typhoid fever is a distinctive acute systemic febrile infection caused by several serotypes of the Gramnegative bacillus Salmonella (primarily Salmonella choleraesuis serotypes typhi and paratyphi). ${ }^{2}$ A study carried out in a hospital in Pakistan showed that nonpregnant women with typhoid fever were more likely to develop complications as compared to the pregnant women. ${ }^{2}$ The authors of that study also derived the hypothesis that disease complications in typhoid fever are related to host immunity and less likely to occur with the immunological changes associated with pregnancy. ${ }^{2}$ Having said this, when complications due to typhoid perforation do occur, the lives of the mother and the foetus are at stake.
Fetal losses, especially when typhoid perforation occurs during the first and second trimester, are high.$^{3}$ Maternal mortality rate is also high and this is probably due to the physiological changes in pregnancy coupled with the stress of typhoid infection and intra-abdominal sepsis. $^{3}$

Surgery is the gold standard for the treatment of typhoid perforation after adequate resuscitation, correction of electrolyte imbalance and commencement of appropriate antibiotic therapy. ${ }^{2}$ Multiple local, regional, and systemic factors can contribute to post-laparotomy wound dehiscence as occurred in our case. ${ }^{4}$ The patient had wound infection with ongoing intra-abdominal sepsis due the gross peritoneal contamination; bowel distension due to a combination of bowel edema, paralytic ileus and a gravid uterus; and severe anaemia with malnutrition. 
There are different modalities available for the management of wound dehiscence post laparotomy, which range from primary repair with sutures/mesh reinforcement, to entero-atmospheric treatment. ${ }^{5}$ However, in the presence of intra-abdominal sepsis and a gradually enlarging uterus, the management option with the best outcome for both mother and foetus becomes more difficult to choose due to fewer viable options. An online search for the management of similar cases revealed 3 cases of burst abdomen during pregnancy, 2 due to incisional hernias, and the third due to surgical site infection post laparotomy. ${ }^{6,7,8}$ The first case had a burst abdomen due to an incisional hernia at a gestational age of 28 weeks which was initially managed with a mesh hernia repair and tension sutures. The baby was subsequently delivered by Caesarean section at 34 weeks with a concurrent surgical repair of the hernia. ${ }^{6}$ The second case of burst abdomen due to an incisional hernia occurred at 35 weeks gestation, and underwent a Caesarean section with concurrent hernia repair without a mesh. ${ }^{7}$ These cases had no overt on-going intra-abdominal sepsis, hence the choice of management. The third case developed an intra-abdominal abscess with wound failure at a gestational age of 27 weeks post small bowel resection, and was managed on Negative Pressure Wound Therapy (NPWT). The wound was healed in 4 weeks, and the baby was delivered vaginally at 39 weeks.

Due to the paucity of literature on the management of wound dehiscence due to intra-abdominal sepsis in pregnant patients, the authors have suggested a management algorithm (Figure 1). It takes a broad look at the management of the burst abdomen in pregnancy according to the presence of intra-abdominal sepsis and the gestational age of viability which is 24 weeks according to WHO guidelines. ${ }^{9}$ Those who present with intra-abdominal sepsis usually undergo an elective incisional hernia repair after management of the pregnancy and the sepsis.

Important points to consider are:

1. The availability of neonatal intensive care units for resuscitation and management of preterm deliveries.

2. Management decisions must be made based on the health of the mother, not of the foetus.

3. Gestational age of viability differs among different countries, depending on the quality of their neonatal intensive care units. The suggested algorithm can be adapted to suit the selected gestational age of viability for whichever health care system it is implemented in.
The advantage of NPWT post wound dehiscence is well documented in literature. ${ }^{4}$ There is no evidence of randomized controlled trials involving use of NPWT in gravid patients. This may be due to the rarity of such cases. However, the two cases in which NPWT were used in gravid patients reported excellent outcomes for both mother and baby. 8,10

\section{CONCLUSION}

Management of the acute abdomen, and the sequelae of complications that may arise in pregnancy is a challenge even for the best equipped facilities. There is a paucity of literature on the management of wound dehiscence in pregnancy with/without intra-abdominal sepsis. A management algorithm for such cases has been suggested.

Although no adverse effects have been reported in the cases in which NPWT was used in the gravid patient, randomized controlled trials and long term follow up would be necessary to confirm its overall safety in pregnancy and absence of long term effects on the foetus. Decisions on management of these cases must be made based on the health of the mother.

\section{REFERENCES}

1. Goran Augustin, Mate Majerovic. Non-obstetric acute abdomen during pregnancy. Eur J Obstet Gynecol Reprod Biol 2007; 131: 4 - 12.

2. Sulaiman K, Sarwari AR; Culture-confirmed typhoid fever and pregnancy. Int J Infect Dis 2007; 11:337-341.

3. Na'aya HU, Eni UE, Chama CM. Typhoid Perforation in Maiduguri, Nigeria. Ann Afr Med 2004; 3(2): $69-72$.

4. Heller L, Levin S, Butler C. Management of abdominal wound dehiscence using vacuum assisted closure in patients with compromised healing. $\mathrm{Am} J$ Surg 2006; 191(2): 165 - 172.

5. Lopez-Cano M, Pereira J, Armengol-Carrasco M. Acute postoperative open abdominal wall: Nosological concept and treatment implications. World $J$ Gastrointest Surg 2013; 5(12):314 -320.

6. Kumar R, Sonika A, Kaberi B, Sunesh K, Charu C. Gravid Uterus in an incisional hernia leading to burst abdomen. The Internet Journal of Gynecology and Obstetrics 2004; 5 (1).

7. Dubey C, Gupta N, Gupta C, Arora R, Sachdeva P. Successful pregnancy outcome in a case of gravid uterus in an incisional hernia leading to burst abdomen. The Internet Journal of Gynecology and Obstetrics 2009; 13 (1).

8. Shapiro S, Mumme D. Use of Negative Pressure Wound Therapy in the Management of Wound De- 


\section{Case report}

hiscence in a Pregnant Patient. Wounds 2008; 20 (2): $46-48$.

9. Ikechebelu JI, Eleje GU, Ugochukwu EF, Edokwe ES. Should we re-define age of Fetal Viability in Nigeria? A Case Report of Newborn Survival from pre-viable pre-labor rupture of membranes. $J$ Womens Health Issues Care 2014; 3:3.
10. Asukai K, Kashiwazaki M, Koizumi K, Nobunaga T, Yano H. A case report of a 19-week gravid patient with a dehisced abdominal wound and treated with V.A.C. ATS $^{\circledR}$ Therapy System. Int Wound J 2014; doi:10.1111/iwj.12352 